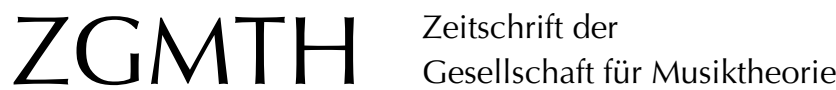

Orgass, Stefan (2011): Musiktheoretische Tätigkeit als Differenzierung musikalischer und musikbezogener Unterscheidungen. ZGMTH 8/1, 27-29.

https://doi.org/10.31751/617

(C) 2011 Stefan Orgass

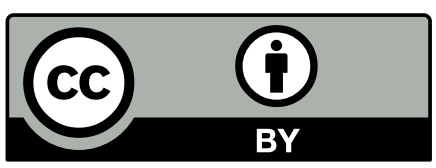

Dieser Text erscheint im Open Access und ist lizenziert unter einer Creative Commons Namensnennung 4.0 International Lizenz.

This is an open access article licensed under a Creative Commons Attribution 4.0 International License.

veröffentlicht / first published: 14/06/2011

zuletzt geändert / last updated: 13/12/2013 


\title{
Musiktheoretische Tätigkeit als Differenzierung musikalischer und musikbezogener Unterscheidungen
}

\author{
Stefan Orgass
}

Was ist für Sie Musiktheorie?

Da ich mich derzeit unter verschiedenen Aspekten mit George Spencer-Browns Unterscheidungstheorie (bzw. Theorie der Form der Unterscheidung) auseinandersetze, versuche ich eine Antwort vor diesem theoretischen Hintergrund. Musiktheorie könnte dann als Disziplin beschrieben werden, in der untersucht und gelehrt wird, welche Unterscheidungen für eine Musik maßgeblich sind und warum sie maßgeblich sind. Der letztgenannte Gesichtspunkt verweist auf Regeln des musikalischen bzw. musikbezogenen Unterscheidens, wobei Regeln zwischen erlaubten und verbotenen Handlungen unterscheiden und sich der Unterschied zwischen musikalisch und musikbezogen auf die Differenz zwischen einem Denken in Musik einerseits (musikalisches Unterscheiden) und dessen Sprachbezug andererseits (musikbezogenes Unterscheiden) bezieht. Mir scheint in letztgenannter Differenz auch ein möglicher Schlüssel für die Beantwortung der Frage vorzuliegen, ob denn Musiktheorie nun eine Kunst oder eine Wissenschaft sei: Soweit sie den letztgenannten Aspekt akzentuiert, könnte man sie als wissenschaftliche Disziplin mit starker Affinität zur Musikwissenschaft begreifen; soweit sie sich darum bemüht, musikalisches Unterscheiden - auf welchem Wege, d. h. in welchen bzw. durch welche Medien musikalischer Hervorbringung auch immer (Notentexte, elektronische Files, Livepräsentationen etc.) - zu thematisieren, wird man sie eher als künstlerische Disziplin mit einer gewissen Nähe zur Komposition betrachten (bzw. beobachten). Diese Zaunreiterposition scheint für Musiktheorie konstitutiv zu sein, also auf die Notwendigkeit eines fortwährend zu prozessierenden Kreuzens der Grenze zwischen Musik- und Sprachbezug - genauer: zwischen dem Kreuzen der Grenzen zwischen musikalischen Einheiten einerseits und zwischen musikalischen Einheiten und ihrer begrifflichen Erfassung bzw. regelbezogenen Einordnung durch Prädikation andererseits - hinzuweisen. Würde die erwähnte Frage eines Tages beantwortet, gäbe es diese Disziplin wohl nicht mehr.

Welche Erfahrungen (z. B. während der eigenen Ausbildung oder in der Hochschule) machen Sie für Ihr Bild von Musiktheorie besonders verantwortlich?

Die oben gegebene Antwort ist sowohl mit meinem eigenen Studium (Lehramt Musik und Musikwissenschaft) als auch mit meinen beruflichen Erfahrungen kompatibel: Sowohl damals als auch in der jetzigen Hochschullehrertätigkeit sind mir Musiktheoretike- 
rinnen und Musiktheoretiker begegnet, die durch ihre Lehrtätigkeit und im Gespräch die erwähnte Zaunreiterposition auf verschiedene Weise bezogen und inhaltlich ausgestaltet haben. In meiner Tätigkeit als Dekan des Fachbereichs 2 unserer Hochschule (2002 bis 2006) habe ich mich - selbstverständlich gemeinsam mit ähnlich gesinnten Kolleginnen und Kollegen - für eine damals anstehende Neubesetzung zweier MusiktheorieProfessuren mit Kollegen eingesetzt, die einen deutlicheren Akzent (als dies vormals zu beobachten gewesen war) auf ein Erlernen musiktheoretischer Begriffe und Regeln bzw. Regelwerke gesetzt haben, welches konsequent von eigener musikalischer Tätigkeit bzw. Praxis ausgeht (bevor dann das unstrittig wichtige sEigenleben der Begriffe und Regeln in den Blick kommen und gepflegt werden kann). Dies ist wohl eine musikpädagogische Sicht auf Musiktheorie, die aber offensichtlich auch musiktheorieimmanent eingenommen werden kann und wird.

\section{Wozu Musiktheorie in der Schulmusikausbildung?}

Zu einem wichtigen Aspekt dieser Frage - dem des Sinns musikalischer Analyse aus der Sicht der von mir vertretenen Kommunikativen Musikdidaktik - habe ich an anderer Stelle bereits umfänglich Stellung genommen. ${ }^{1}$ Dort wird eine bedeutungstheoretische Sicht auf musikalische Analyse entfaltet. `Analyse` wird als Oberbegriff für den Zusammenhang von Analyse und Interpretation verwendet, da es keine interpretations- bzw. 'wertıfreie Analyse gibt. Analyse konkretisiert das interaktive Musiklernen im Musikunterricht in Tätigkeiten, »die a) in normativer Hinsicht auf die Ermöglichung der Auseinandersetzung mit der Vielfalt von Musik und von musikbezogenen Zuweisungen von Bedeutung und Bedeutsamkeit ausgerichtet sind, b) inhaltlich auf die Erweiterung von Möglichkeiten für solche Zuweisungen sowie auf die Gewährleistung von deren stimmigen Zusammenhang und damit auf die Verbesserung musikbezogener Verständigung zielen (wobei die Stimmigkeit auf der Grundlage des jeweils gewählten Aspekts bzw. vor dem Hintergrund der jeweiligen Zielsetzung zu beurteilen ist) und insofern c) dem Musiklernen in seinen vier Dimensionen eine Richtung gibt. $\Perp^{2}$ In einer Musikdidaktik, die bedeutungstheoretisch (und eben nicht erfahrungstheoretisch) ansetzt, kommt also musikalischer Analyse im angedeuteten Sinne ein zentraler Stellenwert zu. Angehende Musiklehrerinnen und Musiklehrer sollten die entsprechenden `Kompetenzen entwickelt haben. Daher gibt es an Folkwang z. B. die Disziplin `Didaktik der Musiktheorie`, welche als »künstlerisches» Fach geführt wird.

\section{Wozu Musiktheorie in der Schule?}

Ein wichtiger Aspekt wurde bereits in der Antwort auf die vorige Frage artikuliert: Musiktheoretische Tätigkeit im erläuterten Sinne ermöglicht neue, bislang durch das Individuum, das diese Tätigkeit ausübt, so (kognitiv) nicht prozessierte musikbezogene Bedeutungszuweisungen (also musikalische Unterscheidungen und Wahrnehmungen sowie Deutun-

1 Orgass 2007.

2 Ebd., $625 \mathrm{f}$. Musiklernen wird hier als Zusammenhang von musikbezogener Wahrnehmung, Deutung, Orientierung und Selbsttätigkeit begriffen. 
gen vom Zusammenhang bzw. von Zusammenhängen des Unterschiedenen) und eröffnet begriffliche Möglichkeiten der Verständigung über diese Bedeutungszuweisungen. In diesem weiten Sinne gibt es Musiktheorie z. B. auch im Grundschulunterricht, denn die - in historischer Hinsicht auszudifferenzierende - musiktheoretische Fachterminologie macht lediglich einen kleinen, wenngleich disziplinär wichtigen Teil dieser begrifflichen Möglichkeiten aus. Allerdings gehören meines Erachtens begründbare Vorstellungen vom Erlernen von Begriffen, auch von musiktheoretischen Fachbegriffen, zur Theoretisierung der letzteren, womit die immanente Didaktizität der Musiktheorie umrissen sein mag. ${ }^{3}$

Vertreten Sie als Musikpädagoge Positionen, von denen Sie behaupten würden, sie verhielten sich komplementär zu dem, wofür Musiktheorie Ihrer Ansicht nach steht?

Nein, unter Berücksichtigung der bislang mit Musiktheoretikerinnen und Musiktheoretikern geführten Gespräche kann ich beobachten, dass die oben angedeuteten Ansichten (etwa zur immanenten Didaktizität der Musiktheorie) in zunehmendem Maße von diesen Gesprächspartnerinnen und Gesprächspartnern geteilt werden.

Wie schätzen Sie das Verhältnis der Disziplinen Musikpädagogik und Musiktheorie in der Hochschullandschaft hierzulande ein?

Für die Beantwortung dieser Frage fehlt mir eine Menge einschlägiger Beobachtungen bzw. Beobachtungsmöglichkeiten. Sicherlich sollten und können beiden Disziplinen aber voneinander lernen: Die Musiktheorie, insofern für sie beispielsweise die oben nur angerissenen Vorstellungen von der Prozesshaftigkeit des Zuweisens von Bedeutungen zu einer Musik (bzw. von dem, was oben immanente Didaktizität der Musiktheorie genannt wurde) nicht selbstverständlich, aber mindestens bedenkenswert sein könnten; die Musikpädagogik, insofern sie beispielsweise die zur Zeit in ihr beobachtbare Tendenz zur `Enthistorisierung`musikunterrichtlicher Inhalte (vgl. etwa den Tonsatz eines Arrangements für das Klassenmusizieren, der - im besten Fall - an die Zeiten des vierstimmigen Satzes erinnert, oder auch die musikdidaktischen Hervorbringungen im Umfeld des so genannten Aufbauenden Musikunterrichts), im Gespräch mit historisch arbeitenden Musiktheoretikerinnen und Musiktheoretikern (kann es eine Alternative zu dieser historischen Arbeitsweise geben?) in Frage stellen und diese aus meiner Sicht unangemessene Bedeutungszuweisung zur Musik relativieren und korrigieren könnten.

\section{Literatur}

Orgass, Stefan (2007), »Musikalische Analyse aus Sicht Kommunikativer Musikdidaktik. Analysieren im Musikunterricht als Differenzierung, Relativierung oder Korrektur musikbezogener Bedeutungszuweisungen«, in: Musikalische Bildung in europäischer Perspektive. Entwurf einer Kommunikativen Musikdidaktik, Hildesheim u. a.: Olms, 605-706.

3 Vgl. ebd.: Musikbezogene Bedeutungszuweisungen werden sowohl im Vorschulalter als auch im musikwissenschaftlichen Doktorandenkolloquium differenziert, relativiert oder korrigiert etc. 